\title{
Measurement Reactivity of Accelerometer-Based Sedentary Behavior and Physical Activity in 2 Assessment Periods
}

\author{
Antje Ullrich, Sophie Baumann, Lisa Voigt, Ulrich John, and Sabina Ulbricht
}

\begin{abstract}
Background: The purposes of this study were to examine accelerometer measurement reactivity (AMR) in sedentary behavior (SB), physical activity (PA), and accelerometer wear time in 2 measurement periods and to quantify AMR as a human-related source of bias for the reproducibility of SB and PA estimates. Methods: In total, 136 participants (65\% women, mean age $=54.6 \mathrm{y}$ ) received 7-day accelerometry at the baseline and after 12 months. Latent growth models were used to identify AMR. Intraclass correlations were calculated to examine the reproducibility using 2-level mixed-effects linear regression analyses. Results: Within each 7-day accelerometry assessment, the participants increased their time spent in SB $(b=2.4$ min/d; $b=3.8 \mathrm{~min} / \mathrm{d})$ and reduced their time spent in light PA $(b=-2.0 \mathrm{~min} / \mathrm{d} ; b=-3.2 \mathrm{~min} / \mathrm{d})$, but did not change moderate to vigorous PA. The participants reduced their wear time $(b=-5.2 \mathrm{~min} / \mathrm{d})$ only at the baseline. The intraclass correlations ranged from .42 for accelerometer wear time to .74 for SB. The AMR was not identified as a source of bias in any regression model. Conclusions: AMR may influence SB and PA estimates differentially. Although 7-day accelerometry seems to be a reproducible measure, our findings highlight accelerometer wear time as a crucial confounder in analyzing SB and PA data.
\end{abstract}

Keywords: accelerometry, physical activity assessment, methods

In order to determine the level of sedentary behavior (SB) and physical activity (PA), to understand their relationship with health, and to evaluate the efficacy of behavioral interventions, an accurate measure of SB and PA is required. ${ }^{1}$ This is challenging because both behaviors are characterized by considerable interindividual and intraindividual variability. ${ }^{2}$ Day-to-day variability around a mean level is a natural part of SB and PA and is described as the habitual level of the SB and PA of an individual. ${ }^{3,4}$ This behavioral variability needs to be carefully studied because it has major implications for the measurement and conclusions drawn from data analysis. ${ }^{4}$

Accelerometry is frequently used to assess SB and PA. Accelerometer-based measures might be subject to human-related sources of bias. ${ }^{5}$ Participants can influence data measurement by changing their behavior when they are aware of wearing an accelerometer. ${ }^{6}$ Social desirability or the motivation to be physically more active than usual due to wearing such a device might be reasons for the presence of accelerometer measurement reactivity (AMR). ${ }^{7}$ So far, evidence on AMR among adults is inconsistent and mostly limited to certain intensities such as moderate to vigorous PA (MVPA), ${ }^{8-14}$ to young adults, or small sample sizes. ${ }^{10-13}$ However, Davis and Loprinzi ${ }^{8}$ have observed that the total activity counts per day from days 2 and 3 differed significantly from the first day of measurement over the measurement period (4-4.5\% change) in adults. ${ }^{8}$ In addition to AMR, Behrens and Dinger ${ }^{11}$ have argued that daily variations can be better explained by differences in PA on weekdays compared with days of the weekend than by AMR. Furthermore, changes in PA

Ullrich, Baumann, Voigt, John, and Ulbricht are with the Department of Preventive Research and Social Medicine, Institute for Community Medicine, University Medicine Greifswald, Greifswald, Germany; and the German Centre for Cardiovascular Research (DZHK), Partner Site Greifswald, Greifswald, Germany. Baumann is also with the Faculty of Medicine, Institute and Policlinic of Occupational and Social Medicine, Technische Universität Dresden, Dresden, Germany. Ullrich (antje.ullrich@med.uni-greifswald.de) is corresponding author. estimates over the measurement period could be explained by systematic changes in accelerometer wear time. ${ }^{15}$ In comparison to other studies, only the study of Baumann et $\mathrm{al}^{14}$ examined the influence of AMR on SB and the entire intensity spectrum of PA, but they referred to the data of one measurement period only. To our knowledge, none of the studies investigating AMR has examined whether and to what extent AMR occurs in more than one measurement period. The effect of AMR on repeated assessments could result in an overestimation or underestimation of SB and PA estimates in either measurement period and, therefore, in difficulties in detecting changes in SB and PA outcome variables in the long term.

The vast majority of studies applied a 7-day accelerometry protocol to determine the habitual levels of SB and PA. ${ }^{16}$ However, this approach raises the question of whether this length of monitoring protocol is sufficient to reflect the mean level on the entire intensity spectrum from SB to MVPA. Reliable estimates depend on the variability within a person's daily activity pattern, ${ }^{3}$ especially for more than one measurement period. ${ }^{17}$ Moreover, the potential impact of AMR on the reproducibility of SB and PA estimates was not investigated so far.

Therefore, the present study has 2 aims: (1) to examine AMR over 2 measurement periods indicated by systematic changes in SB, light PA (LPA), MVPA, and accelerometer wear time, and (2) to quantify whether AMR should be considered as a source of bias in order to estimate the reproducibility of SB and PA data.

\section{Methods}

\section{Study Participants}

A sample of 1165 individuals aged between 40 and 70 years had been recruited in general medical practices, job agencies, or via a health insurance company between June 2012 and December 2013. The individuals were asked to participate in a stepwise cardiopreventive health examination program. Step 1 included a self-administered 
cardiovascular risk screening via tablet computer followed by blood pressure measurements and blood sample taking on an optional base $(\mathrm{n}=706)$, and step 2 comprised a cardiovascular examination program, including cardiopulmonary exercise testing, using a cycle ergometer $(n=582)$.

Of those individuals who agreed to be contacted again for future studies $(\mathrm{n}=1107)$, a random subsample of eligible individuals aged 40-65 years was drawn. A total of 401 persons were contacted and offered participation in a randomized-controlled study that aimed to assess the feasibility of a brief tailored letter intervention to increase PA and to reduce $\mathrm{SB}$ in leisure time. Among those who had been offered study participation, 175 persons agreed to participate. The study was conducted between February 2015 and August 2016. The design and participant flow were described in detail elsewhere. ${ }^{18}$

The SB, LPA, and MVPA were measured with accelerometry at the baseline $(n=175)$ and after 12 months $(n=165,94 \%)$. Two participants at each measurement period were excluded due to missing accelerometer data (excluded, $n=4$ ). In addition, we analyzed data only among those who had worn the accelerometer $\geq 10$ hours per day on $\geq 5$ days, including at least one weekend day (excluded, $\mathrm{n}=25$ ). The final sample size was composed of 136 participants.

\section{Procedure}

For the randomized-controlled study, all participants underwent the following procedure at the baseline and after 12 months:

1. Cardiovascular health program, including blood sample taking and standardized measurement of blood pressure, waist circumference, body weight, and height at the cardiovascular examination center of the University Medicine Greifswald.

2. Self-administered assessments of sociodemographics, SB, and PA.

3. Wearing an accelerometer for 7 consecutive days.

4. Logging working hours in a diary over the monitoring period.

The study participants were instructed to wear the accelerometer on their right hip with an elastic band outside of any water-based activities during the day, starting the day following the cardiovascular health program. All participants were informed that PA would be recorded for 7 days.

After the baseline assessments, the participants were randomized into an assessment-only group $(\mathrm{n}=85)$ or an intervention group $(\mathrm{n}=90)$. Additionally, for all participants, self-administered assessments of SB and PA were conducted at months 1, 3, 4, and 6 after baseline. Only individuals from the intervention group received up to 3 letters tailored to their self-reported SB and PA at months 1,3 , and 4 . The study was approved by the clinical ethical committee of the University Medicine Greifswald (protocol number: BB 002/15a).

\section{Measures}

Accelerometer-based data were collected using a triaxial ActiGraph Model GT3X+ accelerometer (ActiGraph, Pensacola, FL). The accelerometers were initialized at a sampling rate of $100 \mathrm{~Hz}$, and the raw data were integrated into 10-second epochs. Data from the vertical axis were used. For statistical analysis, data from the accelerometers were downloaded and processed using ActiLife software (version 6.13.3; ActiGraph).
Time spent in SB, LPA, MVPA, and wearing the accelerometer was determined by minutes per day. Nonwear time was calculated by the Troiano algorithm and was defined as at least 60 consecutive minutes of zero activity intensity counts, with allowance for $\leq 2$ minutes of counts (counts per minute) between 0 and 100. To identify the time spent in different intensities of PA, we used cut points according to different intensity threshold criteria. ${ }^{19}$ Values $<100$ counts per minute were determined as SB, values between 100 and 2019 counts per minute as LPA, and values $\geq 2020$ counts per minute as MVPA.

Sex, age, and years of school education $(<10$ y/10-11 y $>12$ y) were obtained by a self-administrative questionnaire. In addition, the study group (assessment-only group/intervention group), time (baseline/after $12 \mathrm{mo}$ ), recruitment site (general practice/job center/ health insurance), first day of measurement (weekday/weekend day), ${ }^{20}$ season of data collection (winter/spring/summer), ${ }^{21}$ and average number of working hours ${ }^{4}$ on each day the accelerometer was worn were included as covariates.

\section{Statistical Analyses}

We decided to include data from both study groups, as all participants received almost the same assessment procedure, the feasibility study was not powered to detect differences between the assessment-only group and intervention group, and previously published data revealed that there were no differences in selfreported PA and SB between groups after 12 months. ${ }^{18}$

The SB, LPA, and accelerometer wear time were approximately normally distributed; thus, untransformed values were used for analyses. To account for their right-skewed distributions, MVPA estimates were square root transformed. For all analyses, $P$ values below .05 were considered statistically significant.

Latent growth models were used to investigate AMR for both measurement periods. ${ }^{22}$ In line with Baumann et al, ${ }^{14}$ time spent in SB, LPA, MVPA, and wearing the accelerometer on each of the 7 days of measurement was represented by 7 observed indicators of these continuous latent variables (growth factors). The indicators were regressed on latent growth factors representing trajectories of outcomes over a week. ${ }^{22}$ A maximum likelihood estimator with robust SEs was used. The shape of the growth curves was determined by time scores defined in the measurement model of the growth factors and matched with the observed day number of the measurement week. To specify nonlinear growth curves, an overall change function (eg, linear, quadratic, cubic) was fitted to the sample by adding quadratic and cubic slopes of time scores to the models. Rescaled likelihood ratio tests were used to test whether higher-order functions of time scores and free growth factor variances were required. ${ }^{23}$ Working hours as a time-varying covariate that was specified to predict outcomes at the corresponding day of measurement has been taken into account for all models. Additionally, accelerometer wear time as a time-varying covariate was used in modeling SB and activity outcome variables (LPA and MVPA). Nonzero time trends in the outcomes over the days of measurement would imply reactivity. In the models, the slope factor was freely estimated if appropriate and treated as a reactivity indicator, reflecting the individual average change in outcome over time. Therefore, the factor scores of the outcomes were saved and included as a reactivity indicator in further analysis. Statistical analyses were performed using Mplus (version 7.316). ${ }^{23}$

To calculate the slope effect size $(d)$ of each outcome variable, we used the following formula: $d=$ mean of the slope/(variance of the slope $\left.{ }^{\wedge} .5\right) .{ }^{24}$ To examine whether reactivity was a consistent 
phenomenon at the within-person perspective, we calculated whether the individuals had the same direction of their individual slopes. Therefore, we compared the values of the slopes of the outcomes (minutes per day) over the measurement period of the baseline with the 7-day assessment period of 12 months apart by creating 2 categories $(1=$ same direction of values of the slopes at both measurement periods $/ 0=$ different direction of values of the slopes at both measurement periods).

For each outcome, the average of the 7 days of measurement was calculated. Two-level (individual and time) mixed-effects linear regression analyses were performed to assess changes in accelerometer-based outcomes from the baseline to 12 months apart, including a random intercept for the participants. All regression models were adjusted for sex, age, education, study group, time, recruitment site, first day of measurement, and season of data collection. In addition, we added the individual average value of accelerometer wear time, the reactivity indicator of the respective SB and PA outcome, and a combination of these factors as potential covariates step by step.

We used intraclass correlation coefficients (ICC) to decide which model for each outcome was most appropriate. The ICC is a measure of reproducibility of replicate measures from the same participant. ${ }^{25}$ The ICC coefficient is classified as follows: less than .40 , poor; between .40 and .75 , fair to good; and .75 or more, excellent reproducibility. ${ }^{25}$ To illustrate the agreement between both measurement periods and to estimate the $95 \%$ limits of agreement, Bland-Altman plots were applied. Statistical analyses were performed using Stata/SE (version 14.2). ${ }^{26}$

\section{Results}

\section{Sample Characteristics}

In our sample, the mean age was 54.6 years, and $65.4 \%$ were women. The majority of the participants attended school for 10 11 years $(70.4 \%)$. Table 1 provides estimates on the time spent in SB, LPA, and MVPA and wearing the accelerometer from the baseline to 12 months. On an accelerometer wearing day, the participants spent most of their time in SB (baseline: 602.6 [96.6] $\mathrm{min} / \mathrm{d}, 68.3 \%$; after $12 \mathrm{mo:} 593.8$ [99.2] $\mathrm{min} / \mathrm{d}, 68.6 \%$ ) or in LPA (baseline: 230.9 [60.1] $\mathrm{min} / \mathrm{d}, 26.2 \%$; after 12 mo: 227.5 [57.9] $\mathrm{min} / \mathrm{d}, 26.4 \%$ ), with little time spent in MVPA (baseline: 47.2 [24.4] $\mathrm{min} / \mathrm{d}, 5.5 \%$; after $12 \mathrm{mo:} 43.5$ [23.0] $\mathrm{min} / \mathrm{d}, 5.0 \%)$. At the baseline $(\mathrm{n}=173), 121$ individuals wore the accelerometer for 7 valid days (69.9\%), 28 for 6 valid days (16.2\%), and 5 for 5 valid days $(2.9 \%)$. After 12 months $(\mathrm{n}=163), 110$ individuals wore the accelerometer for 7 valid days $(67.5 \%), 28$ for 6 valid days (17.2\%), and 7 for 5 valid days $(4.3 \%)$.

Table 1 Descriptive Characteristics of the Study Sample $(n=136)$

\begin{tabular}{|c|c|c|}
\hline Variables & Baseline & 12 months $^{a}$ \\
\hline \multicolumn{3}{|l|}{ Sociodemographic variables } \\
\hline Age, mean (SD), y & $54.6(6.3)$ & \\
\hline \multicolumn{3}{|l|}{ Sex, n $(\%)$} \\
\hline Women & $89(65.4)$ & \\
\hline \multicolumn{3}{|l|}{ School education, $\mathrm{n}(\%), \mathrm{y}$} \\
\hline$<10$ & $7(5.2)$ & \\
\hline $10-11$ & $95(70.4)$ & \\
\hline$\geq 12$ & $33(24.4)$ & \\
\hline \multicolumn{3}{|l|}{ Variables related to data collection } \\
\hline \multicolumn{3}{|l|}{ Recruitment site, n (\%) } \\
\hline General practice & $54(39.7)$ & \\
\hline Job center & $24(17.6)$ & \\
\hline Health insurance company & $58(42.7)$ & \\
\hline \multicolumn{3}{|l|}{ First day of measurement, n (\%) } \\
\hline Weekday & $113(83.1)$ & $107(78.7)$ \\
\hline \multicolumn{3}{|l|}{ Season, n $(\%)$} \\
\hline Winter & $7(5.2)$ & $22(16.2)$ \\
\hline Spring & $120(88.2)$ & $86(63.2)$ \\
\hline Summer & $9(6.6)$ & $28(20.6)$ \\
\hline Working hours, mean (SD), h/d & $4.1(3.0)$ & $3.5(3.0)$ \\
\hline \multicolumn{3}{|l|}{ Accelerometer-based variables, mean (SD) } \\
\hline Accelerometer wear time, $\mathrm{min} / \mathrm{d}$ & $880.8(85.9)$ & $864.8(93.6)$ \\
\hline Sedentary behavior, $\mathrm{min} / \mathrm{d}$ & $602.6(96.6)$ & $593.8(99.2)$ \\
\hline Light physical activity, min/d & $230.9(60.1)$ & $227.5(57.9)$ \\
\hline Moderate to vigorous physical activity, $\mathrm{min} / \mathrm{d}$ & $47.2(24.4)$ & $43.5(23.0)$ \\
\hline Sedentary behavior, $\%$ of wearing accelerometer & $68.3(7.9)$ & $68.6(7.7)$ \\
\hline Light physical activity, $\%$ of wearing accelerometer & $26.2(6.5)$ & $26.4(6.6)$ \\
\hline Moderate to vigorous physical activity, $\%$ of wearing accelerometer & $5.5(3.1)$ & $5.0(2.6)$ \\
\hline
\end{tabular}

${ }^{\mathrm{a}}$ One participant with missing values on 1 day of accelerometer-based variables $(\mathrm{n}=135)$. 


\section{Accelerometer Measurement Reactivity}

As shown in Figure 1, the participants increased their time spent in SB by 2.4 minutes per day $(P=.033$; overall increase per week: $14.1 \mathrm{~min} /$ $w k)$ and reduced their time spent in LPA by 2.0 minutes per day $(P=.033$; overall decrease per week: $11.3 \mathrm{~min} / \mathrm{wk})$ at the baseline. After 12 months, the participants increased their time spent in SB by 3.8 minutes per day $(P=.001$; overall increase per week: $23.1 \mathrm{~min} /$ wk) and reduced their time spent in LPA by 3.2 minutes per day
( $P=.001$; overall decrease per week: $19.4 \mathrm{~min} / \mathrm{wk}$ ) over the 7-day wearing period. Time spent in MVPA did not change significantly at the baseline $(\beta=-0.00 ; P=.701$; overall decrease per week: $0.0 \mathrm{~min} /$ wk) or after 12 months $(\beta=-0.00 ; P=.165$; overall decrease per week: $0.1 \mathrm{~min} / \mathrm{wk})$. The participants significantly reduced the accelerometer wear time by 5.2 minutes per day $(P=.030$; overall decrease per week: $31.1 \mathrm{~min} / \mathrm{wk}$ ) at the baseline. After 12 months, the participants did not significantly change accelerometer wear time $(\beta=-0.20 ; P=.935$; overall decrease per week: $1.2 \mathrm{~min} / \mathrm{wk})$.

\section{Baseline}

A Minutes of sedentary behavior

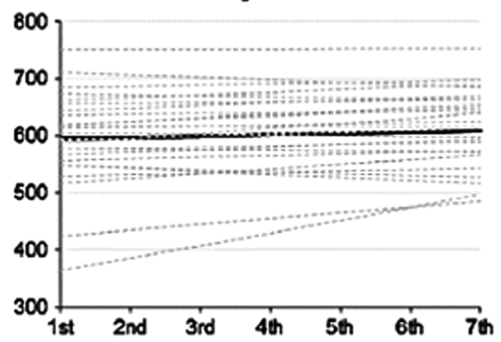

B Minutes of light physical activity

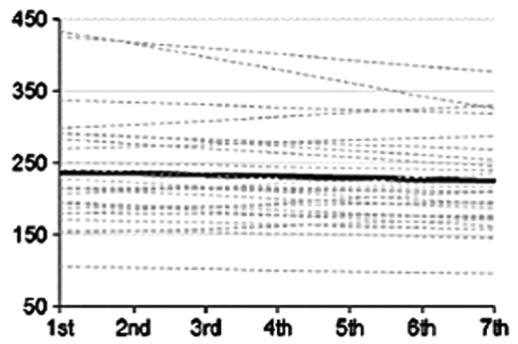

C Minutes of MVPA

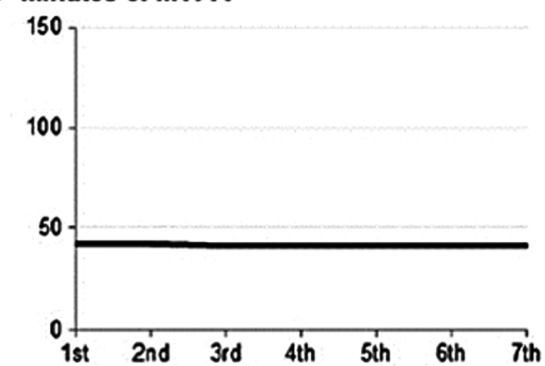

D Minutes of accelerometer wear time

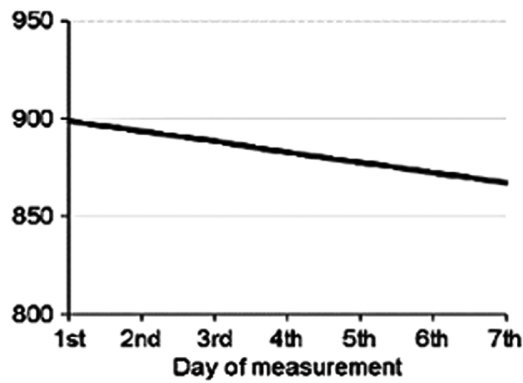

After 12 months
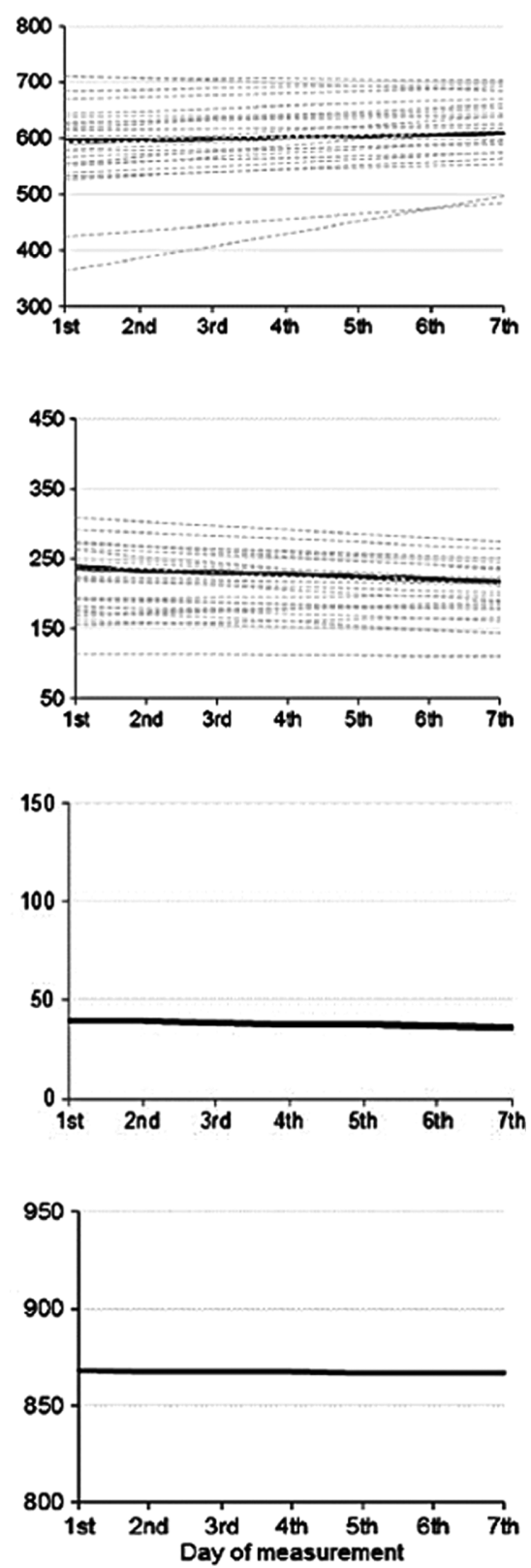

Figure 1 - Estimated average (thick black line) and individual-specific (thin dotted gray lines) linear growth curves for minutes spent in SB (A), light physical activity (B), MVPA (C), and wearing the accelerometer (D) at baseline and after 12 months. All models were adjusted for accelerometer wear time and working hours if appropriate. Note: Individual-specific regression lines of light physical activity and sedentary behavior shown for 25 participants randomly selected from the study sample (20\%). Growth mixture modeling revealed no results for minutes per day of MVPA and accelerometer wear time when linear slope variances were freely estimated. Therefore, no individual-specific regression lines of MVPA and accelerometer wear time can be presented. MVPA indicates moderate to vigorous physical activity. 
Results of the parameter estimates at the baseline and after 12 months for latent growth models of SB, LPA, MVPA, and accelerometer wear time are shown in Supplementary Tables S1 and S2 (available online).

The reactivity indicators of SB $(P=.011)$ and LPA $(P=.008)$ significantly differed from the baseline to 12 months apart. At the within-person perspective, $60.7 \%$ and $64.4 \%$ of the individuals showed slope values of SB $(n=82)$ and LPA $(n=87)$ with the same direction at both measurement points, respectively, but $39.3 \%$ $(n=53 ; \mathrm{SB})$ and $35.6 \%(n=48$; LPA $)$ showed a different behavior over the 7-day assessment at the baseline compared with the other measurement period (after $12 \mathrm{mo}$ ).

\section{Reproducibility of Accelerometer-Based Outcomes}

The ICC $(95 \% \mathrm{CI})$ ranged from fair to good reproducibility of the outcomes; the coefficient values were between .42 (.32 to .53) for accelerometer wear time and .74 (.68 to .79) for SB (see Supplementary Table S3 [available online]). The regression model additionally adjusted for the average value of accelerometer wear time was most appropriate for SB $(\mathrm{ICC}=.74)$, LPA $(\mathrm{ICC}=.69)$, and MVPA $(\mathrm{ICC}=.70)$. For accelerometer wear time, the basic model was used $(\mathrm{ICC}=.42)$. Reactivity was not identified as a source of bias in any regression model.

The Bland-Altman plots visualize the agreement between the 2 measurement periods as a function of the mean of these 2 measurement periods (see Supplementary Figure S1 [available online]). The plots showed that the baseline values in all outcome variables were higher than after 12 months. The mean difference (baseline - after $12 \mathrm{mo}$ ) of both measurements of SB was $8.8 \mathrm{~min}$ utes per day $(\mathrm{SD}=87.9 \mathrm{~min} / \mathrm{d})$, LPA was 3.5 minutes per day $(\mathrm{SD}=$ $46.9 \mathrm{~min} / \mathrm{d}$ ), MVPA was 3.7 minutes per day $(\mathrm{SD}=17.3 \mathrm{~min} / \mathrm{d})$, and accelerometer wear time was 16.0 minutes per day $(\mathrm{SD}=$ $95.1 \mathrm{~min} / \mathrm{d})$.

Using the most appropriate regression model for each outcome, neither the time spent in SB, LPA, MVPA, nor the accelerometer wear time significantly declined or increased over time (see Table 2). In all SB and PA regression models, the average value of accelerometer wear time was a significant confounder.

\section{Discussion}

The present study has 2 main findings. First, there was a significant linear trend in the SB and LPA time series as an indicator of AMR for the baseline and 12 months apart, whereas MVPA does not seem to be affected by AMR. Furthermore, the systematic changes within accelerometer wear time differed between the 2 measurement periods. Second, our results showed that the time spent in SB, LPA, MVPA, and wearing the accelerometer are fairly stable between the 2 measurement periods. AMR operationalized by a reactivity indicator could not be identified as a human-related source of bias for the estimation of the reproducibility of the SB and PA data.

In line with previous literature, the results of the present study indicate that persons change SB and PA in the presence of an accelerometer.9,10,12-14 This study adds to the literature by showing that AMR differentially influences SB and PA in 2 measurement periods depending on the intensity level of PA. As shown by Baumann, ${ }^{14}$ individuals seemed to be replacing LPA by SB in one measurement period. LPA includes standing and walking at a light pace, and therefore, SB and LPA are highly correlated. ${ }^{27,28}$ In addition, our study shows that the trend of both time series seems to be the same over the 2 measurement periods. Therefore, it can be assumed that AMR has a similar effect on SB and LPA in 2 measurement periods, 12 months apart. The best advice for reducing bias due to AMR is to consider this in the planning of the study. ${ }^{29}$ In the case of interventions, measurement periods in terms of duration and frequency, as well as number of methods used to assess SB and PA, should be balanced to identify potential intervention effects. To increase the accuracy of the measurement, a longer measurement period may be reasonable. Although a longer measurement period might improve reproducibility, the burden for study participants and study feasibility should be considered because it influences the response rate and compliance. ${ }^{30}$ In our study, we have not been able to draw any conclusions about the "real" size and duration of the AMR because the true SB and PA level is unknown. Therefore, further research is required to determine the size up to which AMR can be ignored.

Although the effect sizes of the changes of the slopes of SB (baseline: $d=0.30$; after $12 \mathrm{mo}: d=0.50$ ) and LPA (baseline: $d=0.29$; after 12 mo: $d=0.54$ ) over one measurement period were small to medium, the validity of the habitual levels of accelerometer-derived estimates of time spent in SB and LPA could be biased due to participant behavior. The results of the in-depth examination of individuals' values of the slopes of SB and LPA in comparison with the baseline and 12-month follow-up showed that almost $40 \%$ (SB) and 35\% (LPA) of individuals changed their reactive behavior from one monitoring period to the other. Although

\section{Table 2 Results of Multilevel Mixed-Effects Linear Regression Models for Minutes of SB, LPA, MVPA, and Accelerometer Wear Time $(n=136)$}

\begin{tabular}{|c|c|c|c|c|c|c|c|c|}
\hline \multirow[b]{2}{*}{ Predictors } & \multicolumn{2}{|l|}{ SB } & \multicolumn{2}{|l|}{ LPA } & \multicolumn{2}{|l|}{ MVPA } & \multicolumn{2}{|c|}{$\begin{array}{c}\text { Accelerometer wear } \\
\text { time }\end{array}$} \\
\hline & b $(95 \% \mathrm{Cl})$ & $P$ & b $(95 \% \mathrm{Cl})$ & $P$ & b $(95 \% \mathrm{Cl})$ & $P$ & b $(95 \% \mathrm{CI})$ & $P$ \\
\hline $\begin{array}{l}\text { Study group (Ref. assessment-only } \\
\text { group) }\end{array}$ & $\begin{array}{c}19.68 \\
(-2.92 \text { to } 42.29)\end{array}$ & .088 & $\begin{array}{c}-20.06 \\
(-38.80 \text { to }-1.31)\end{array}$ & .036 & $\begin{array}{c}0.16 \\
(-0.51 \text { to } 0.54)\end{array}$ & .953 & $\begin{array}{c}7.93 \\
(-22.21 \text { to } 38.07)\end{array}$ & .606 \\
\hline Time (Ref. baseline) & $\begin{array}{c}3.93 \\
(-8.38 \text { to } 16.25)\end{array}$ & .531 & $\begin{array}{c}0.30 \\
(-10.69 \text { to } 11.29)\end{array}$ & .957 & $\begin{array}{c}-0.29 \\
(-0.59 \text { to } 0.01)\end{array}$ & .062 & $\begin{array}{c}-20.13 \\
(-43.81 \text { to } 3.54)\end{array}$ & .096 \\
\hline Study group $\times$ time & $\begin{array}{c}-6.23 \\
(-22.63 \text { to } 10.17)\end{array}$ & .457 & $\begin{array}{c}1.19 \\
(-13.50 \text { to } 15.89)\end{array}$ & .874 & $\begin{array}{c}0.42 \\
(0.01 \text { to } 0.82)\end{array}$ & .044 & $\begin{array}{c}9.0 \\
(-23.41 \text { to } 41.33)\end{array}$ & .588 \\
\hline Accelerometer wear time & $\begin{array}{c}0.76 \\
(0.69 \text { to } 0.83)\end{array}$ & $<.001$ & $\begin{array}{c}0.19 \\
(0.13 \text { to } 0.26)\end{array}$ & $<.001$ & $\begin{array}{c}0.00 \\
(0.00 \text { to } 0.01)\end{array}$ & $<.001$ & - & \\
\hline
\end{tabular}

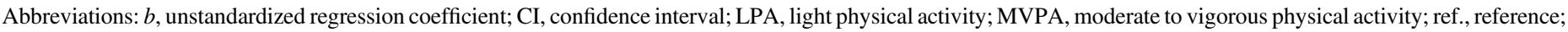

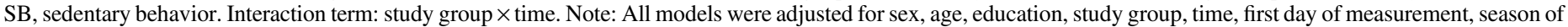
data collection, and recruitment site. Furthermore, models of SB, LPA, and MVPA were adjusted for accelerometer wear time. 
the mean values of the indicators of reactivity of SB and LPA showed the same direction and magnitude in both periods, these results showed that reactivity might be an important human-related source of bias for SB and LPA at the within-person perspective in planning or evaluating the results of longitudinal studies and intervention studies. AMR could interact with interventions by masking their potential effects, particularly in behavioral intervention studies that typically expect small-to-medium effect sizes.

In addition, there is no consensus in the literature about how many days are relevant to achieve a reliable measurement of habitual levels of SB and $\mathrm{PA}^{31}$ and over what period of time reactivity seems to last. In order to underpin the last argument, we have examined whether there is evidence of reactivity if the first day of wearing the accelerometer was excluded. This sensitivity analysis yielded similar results. In the literature, there are different recommendations of monitoring regimes, including different lengths of familiarization periods, ranging from 2 days to 7 days. ${ }^{7}$ Therefore, to what extent AMR may result in an overestimation or underestimation of SB or LPA remains to be investigated.

Furthermore, our results showed that MVPA seemed to be less altered by AMR than SB and LPA. This could be because MVPA typically requires more planning and is likely to be more structured than lighter physical activities. However, it has been argued that MVPA is less predictable on a day-to-day basis and requires longer monitoring periods to determine reproducible habitual behavior. ${ }^{3,4}$ In line with our results, a study that examined the reproducibility in accelerometer-assessed SB and PA reported the highest ICC values for $\mathrm{SB} .{ }^{17}$ It should be noted that ICC coefficients are restricted by the sample in which it was collected because the magnitudes of intraindividual and interindividual variability in SB and PA depend on the characteristics of the study sample. ${ }^{30}$ Although the results reported in this study indicate that a 7-day accelerometry monitoring seems to be a reproducible measure of SB and PA, BlandAltman plots showed that there is a high intraindividual variability in SB and PA estimates. Therefore, the findings should be interpreted with caution, and future studies are required to verify the findings of the present study using a larger sample of adults.

Our findings on accelerometer wear time highlight the importance of this factor. The value for the ICC coefficient of accelerometer wear time was the lowest of all outcomes. In terms of AMR, the systematic changes in time of wearing the accelerometer differed in the magnitude between the repeated measurements. After 12 months, the participants did not significantly change accelerometer wear time. The fact that individuals have become familiar with the monitoring regime and wear the accelerometer more consistently at the second measurement period could partially explain this result. Furthermore, accelerometer wear time was the only relevant predictor in all SB and PA regression models, whereas AMR operationalized by a reactivity indicator could not be identified as a human-related source of bias for the reproducibility of PA and SB estimates. In line with other studies, this indicates that accelerometer wear time should be considered as a crucial confounder in the data analysis of accelerometer data because it may influence potential interventional effects in different ways over time. ${ }^{15,30}$ In order to minimize measurement bias due to systematic variations of accelerometer wear time and therefore to improve the data analysis of SB and PA, some efforts have been made that go beyond simply taking into account the daily time spent wearing the accelerometer. For example, these include developing a standardization methodology 32 or using methods of imputing accelerometer nonwear time. ${ }^{33}$

Some limitations have to be discussed. First, generalizability of our results may be compromised due to selection bias.
The proportion of individuals who declined to participate was $44 \%$. Nonparticipation could reduce study sample representativeness, ${ }^{34}$ in the way that individuals who are more interested in their health or motivated to change their behavior are also more likely to participate. ${ }^{35}$ In line with this finding, our sample seems to be compliant with an average accelerometer wear time of $\geq 14$ hours per day. As reactivity may be most pronounced in people with a high motivation to change their behavior, AMR may have been overestimated in our study. Second, we used hip-worn accelerometers that cannot differentiate between sitting and standing still because movement is determined by acceleration rather than body posture. ${ }^{36}$ Thus, our findings regarding SB can give a distorted picture of AMR. Third, the inclusion of the intervention and assessment-only groups in the analysis might have caused additional variation to the data, as the intervention group may (differentially) change their habitual SB and PA level over time. Unfortunately, our analyses of AMR, separated by the assessment-only group and intervention group, revealed no representative results due to their small sample sizes. Therefore, future studies are required to examine how intervention and control groups differ with regard to AMR using a larger sample. Finally, using the slope factor as a reactivity indicator to operationalize AMR is just one of several other ways to account for AMR for estimating the reproducibility of SB and PA data.

In conclusion, the results of the present study show that AMR differentially influences SB and PA in 2 measurement periods, depending on the intensity level of PA. Although a 7-day accelerometry monitoring seems to be a reproducible measure of SB and PA, our findings highlight the importance of accelerometer wear time as a crucial confounder when using an accelerometer in monitoring SB and PA or planning interventions.

\section{Acknowledgments}

The authors wish to thank the funders, supporters, and participants of the study. This study was funded by the Federal Ministry of Education and Research as part of the German Centre of Cardiovascular Research, DZHK (grant no. 81/Z540100152). The DZHK had no direct role in the development of the methodology; the acquisition, analysis, and interpretation of the data; or in writing the manuscript. All procedures performed in studies involving human participants were in accordance with the ethical standards of the institutional and/or national research committee and with the 1964 Helsinki Declaration and its later amendments or comparable ethical standards. This study was approved by the clinical ethical committee of the University Medicine Greifswald, Germany (protocol number: BB 002/ 15a) and retrospectively registered to the ClinicalTrials.gov Protocol Registration and Results System (clinical trial registration number: NCT02990039; registration date: December 12, 2016). Informed written consent was obtained from all individual participants included in the study. The authors declare that they have no competing interests.

\section{References}

1. De Vries SI, Van Hirtum HW, Bakker I, Hopman-Rock M, Hirasing RA, Van Mechelen W. Validity and reproducibility of motion sensors in youth: a systematic update. Med Sci Sports Exerc. 2009;41(4):818827. doi:10.1249/MSS.0b013e31818e5819

2. Baranowski T, Masse LC, Ragan B, Welk G. How many days was that? We're still not sure, but we're asking the question better! Med Sci Sports Exerc. 2008;40(suppl):S544-S549. doi:10.1249/MSS. 0b013e31817c6651 
3. Bergman P. The number of repeated observations needed to estimate the habitual physical activity of an individual to a given level of precision. PLoS One. 2018;13(2):e0192117. doi:10.1371/journal. pone.0192117

4. Pedersen ES, Danquah IH, Petersen CB, Tolstrup JS. Intra-individual variability in day-to-day and month-to-month measurements of physical activity and sedentary behaviour at work and in leisure-time among Danish adults. BMC Public Health. 2016;16(1):1222. doi:10. 1186/s12889-016-3890-3

5. Pedisic Z, Bauman A. Accelerometer-based measures in physical activity surveillance: current practices and issues. Br J Sports Med. 2015;49(4):219-223. doi:10.1136/bjsports-2013-093407

6. Vanhelst J, Beghin L, Drumez E, Coopman S, Gottrand F. Awareness of wearing an accelerometer does not affect physical activity in youth. BMC Med Res Methodol. 2017;17(1):99. doi:10.1186/s12874-0170378-5

7. Dossegger A, Ruch N, Jimmy G, et al. Reactivity to accelerometer measurement of children and adolescents. Med Sci Sports Exerc. 2014;46(6):1140-1146. doi:10.1249/MSS.0000000000000215

8. Davis RE, Loprinzi PD. Examination of accelerometer reactivity among a population sample of children, adolescents, and adults. $J$ Phys Act Health. 2016;13(12):1325-1332. doi:10.1123/jpah.2015-0703

9. van Sluijs EM, van Poppel MN, Twisk JW, van Mechelen W. Physical activity measurements affected participants' behavior in a randomized controlled trial. J Clin Epidemiol. 2006;59(4):404-411. doi:10.1016/j.jclinepi.2005.08.016

10. Motl RW, McAuley E, Dlugonski D. Reactivity in baseline accelerometer data from a physical activity behavioral intervention. Health Psychol. 2012;31(2):172-175. doi:10.1037/a0025965

11. Behrens TK, Dinger MK. Motion sensor reactivity in physically active young adults. Res $Q$ Exerc Sport. 2007;78(2):1-8. doi:10. 1080/02701367.2007.10599397

12. Eisenberg MH, Phillips LA, Fowler L, Moore PJ. The impact of e-diaries and accelerometers on young adults' perceived and objectively assessed physical activity. Psychol Sport Exerc. 2017;30: 55-63. doi:10.1016/j.psychsport.2017.01.008

13. Vandoni M, Correale L, Del Bianco M, Marin L, Codrons E. Does reactivity to accelerometers occur in a single trial? Brief report in a sample of young adults. J Health Psychol. 2017;22(11):1458-1462. doi:10.1177/1359105316628758

14. Baumann S, Gross S, Voigt L, et al. Pitfalls in accelerometer-based measurement of physical activity: the presence of reactivity in an adult population. Scand J Med Sci Sports. 2018;28(3):1056-1063. doi:10.1111/sms.12977

15. Herrmann SD, Barreira TV, Kang M, Ainsworth BE. Impact of accelerometer wear time on physical activity data: a NHANES semisimulation data approach. Br J Sports Med. 2014;48(3):278282. doi:10.1136/bjsports-2012-091410

16. Migueles JH, Cadenas-Sanchez C, Ekelund U, et al. Accelerometer data collection and processing criteria to assess physical activity and other outcomes: a systematic review and practical considerations. Sports Med. 2017;47(9):1821-1845. doi:10.1007/s40279-0170716-0

17. Kozey-Keadle S, Shiroma EJ, Kamada M, Matthews CE, Harris TB, Lee IM. Reproducibility of accelerometer-assessed physical activity and sedentary time. Am J Prev Med. 2017;52(4):541-548. doi:10. 1016/j.amepre.2016.11.010

18. Voigt L, Baumann S, Ullrich A, Weymar F, John U, Ulbricht S. The effect of mere measurement from a cardiovascular examination program on physical activity and sedentary time in an adult population. BMC Sports Sci Med Rehabil. 2018;10(1):1. doi:10.1186/s13102-018-0090-8
19. Troiano RP, Berrigan D, Dodd KW, Masse LC, Tilert T, McDowell M. Physical activity in the United States measured by accelerometer. Med Sci Sports Exerc. 2008;40(1):181-188. doi:10.1249/mss. 0b013e31815a51b3

20. McVeigh JA, Winkler EA, Howie EK, et al. Objectively measured patterns of sedentary time and physical activity in young adults of the Raine study cohort. Int J Behav Nutr Phys Act. 2016;13(1):41. doi:10. 1186/s12966-016-0363-0

21. Tucker P, Gilliland J. The effect of season and weather on physical activity: a systematic review. Public Health. 2007;121(12):909-922. doi:10.1016/j.puhe.2007.04.009

22. Wang J, Wang X. Structural Equation Modeling: Applications Using Mplus. Chichester, UK: John Wiley \& Sons; 2012.

23. Muthen LK, Muthen BO. Mplus User's Guide. Los Angeles, CA: Muthén \& Muthén; 1998-2012.

24. Brandmaier AM, von Oertzen T, Ghisletta P, Lindenberger U, Hertzog C. Precision, reliability, and effect size of slope variance in latent growth curve models: implications for statistical power analysis. Front Psychol. 2018;9:294. doi:10.3389/fpsyg.2018.00294

25. Rosner B. Fundamentals of Biostatistics. 8th ed. Belmont, CA: Duxbury Press; 2016.

26. Stata Statistical Software: Release 14 [computer program]. College Station, TX: StataCorp LP; 2015.

27. Mansoubi M, Pearson N, Biddle SJH, Clemes S. The relationship between sedentary behaviour and physical activity in adults: a systematic review. Prev Med. 2014;69:28-35. doi:10.1016/j.ypmed. 2014.08.028

28. van der Ploeg HP, Hillsdon M. Is sedentary behaviour just physical inactivity by another name? Int J Behav Nutr Phys Act. 2017;14(1): 142. doi:10.1186/s12966-017-0601-0

29. French DP, Sutton S. Reactivity of measurement in health psychology: how much of a problem is it? What can be done about it? Br J Health Psychol. 2010;15(3):453-468. doi:10.1348/135910710X492341

30. Aadland E, Ylvisaker E. Reliability of objectively measured sedentary time and physical activity in adults. PLoS One. 2015;10(7): e0133296. doi:10.1371/journal.pone.0133296

31. Ricardo LIC, Wendt A, Galliano LM, et al. Number of days required to estimate objectively measured physical activity constructs in different age groups: Findings from three Brazilian (Pelotas) population-based birth cohorts. PLoS One. 2019;15(1):e0216017. doi:10. 1371/journal.pone.0216017

32. Katapally TR, Muhajarine N. Towards uniform accelerometry analysis: a standardization methodology to minimize measurement bias due to systematic accelerometer wear-time variation. J Sci Med. 2014;13(2):379-386.

33. Borgundvaag E, McIsaac M, Borghese MM, Janssen I. Imputing accelerometer nonwear time when assessing moderate to vigorous physical activity in children. $J$ Phys Act Health. 2017;14(11):852860. doi:10.1123/jpah.2016-0706

34. Attwood S, Morton KL, Mitchell J, Van Emmenis M, Sutton S. Reasons for non-participation in a primary care-based physical activity trial: a qualitative study. BMJ Open. 2016;6(5):e011577. doi:10.1136/bmjopen-2016-011577

35. Hardcastle SJ, Hancox J, Hattar A, Maxwell-Smith C, ThogersenNtoumani C, Hagger MS. Motivating the unmotivated: how can health behavior be changed in those unwilling to change? Front Psychol. 2015;6:835. doi:10.3389/fpsyg.2015.00835

36. Heesch KC, Hill RL, Aguilar-Farias N, van Uffelen JGZ, Pavey T. Validity of objective methods for measuring sedentary behaviour in older adults: a systematic review. Int J Behav Nutr Phys Act. 2018;15(1):119. doi:10.1186/s12966-018-0749-2 